\title{
Ionospheric conductivity dependence of dayside region-0, 1, and 2 field-aligned current systems: statistical study with DMSP-F7
}

\author{
K. Haraguchi ${ }^{1}$, H. Kawano ${ }^{1}$, K. Yumoto ${ }^{2}$, S. Ohtani ${ }^{3}$, T. Higuchi ${ }^{4}$, and G. Ueno ${ }^{4}$ \\ ${ }^{1}$ Department of Earth and Planetary Sciences, Kyushu University, 6-10-1 Hakozaki, Fukuoka 812-8581, Japan \\ ${ }^{2}$ Space Environment Research Center, Kyushu University, 6-10-1 Hakozaki, Fukuoka 812-8581, Japan \\ ${ }^{3}$ The Johns Hopkins University Applied Physics Laboratory, Johns Hopkins Road, Laurel, MD 20723-6099, USA \\ ${ }^{4}$ The Institute of Statistical Mathematics, 4-6-7 Minami-Azabu, Minato-ku, Tokyo 106-8569, Japan
}

Received: 21 March 2003 - Revised: 28 December 2003 - Accepted: 14 April 2004 - Published: 7 September 2004

\begin{abstract}
The present study statistically examines the dependence of the intensities of dayside (MLT $=8-12 \mathrm{~h}$ ) large-scale field-aligned currents (FACs) on the ionospheric conductance using the summary data of DMSP-F7 constructed by the procedure of Higuchi and Ohtani (2000). We have found that, in the dayside region, R1 and R0 have a higher correlation between ionospheric conductivity and FAC intensity than R2, suggesting that R0 and R1 are driven by a more voltage-like source than R2. This result is consistent with the idea that $\mathrm{R} 1$ and $\mathrm{R} 0$ are driven by the interaction between the solar wind and the open magnetospheric magnetic field. We have also found that dayside FAC intensities are latitudinally well balanced when they have a three sheet structure (R0, R1 and R2); on the other hand, for a two sheet structure (R1 and R2), the intensity of R1 is larger than that of R2, so that the net current has the polarity of R1.
\end{abstract}

Key words. Ionosphere (Ionosphere-magnetosphere interaction; Polar ionosphere) - Magnetospheric physics (current systems)

\section{Introduction}

Large-scale field-aligned currents (FACs) play an important part in the coupling between the magnetosphere and the ionosphere, thus it is important to understand the mechanism of FACs self-consistently. FACs are classified into three systems according to their polarities and distributions, that is, Region 2 (R2), Region 1 (R1), and Region 0 (R0, also known as cusp current) systems from equatorward to poleward (Iijima and Potemra, 1978). The R2 current flows away from the ionosphere in the prenoon sector and into the ionosphere in the postnoon sector. The R1 current flows in the direction opposite to the R2 current at a given local time. The R0 current is confined in the midday sector and its polarity is the

Correspondence to: $\mathrm{H}$. Kawano

(hkawano@geo.kyushu-u.ac.jp) opposite to that of the R1 current, or the same as that of the R2 current.

The source regions of the three FACs are generally thought to be different: Stern (1984) suggested that the interaction between the solar wind and the magnetosphere at the high-latitude boundary layer (HLBL) generates voltage for driving the R1 current circuit flowing in HLBL. Siscoe et al. (1991) modeled the coupling between HLBL and the low-latitude boundary layer (LLBL) and suggested that the interaction between LLBL and HLBL is an important factor in determining the FAC distribution. Ohtani et al. (1995) proposed, based on particle observations by Viking and DMSP-F7, that R0 and R1 are mapped to HLBL and LLBL, respectively, and that they are closely coupled to the same source. It is noted that this model by Ohtani et al. is consistent with an earlier work on R0 by Erlandson et al. (1988), who suggested from particle observations of VIKING that R0 (which they called the traditional cusp current) maps to HLBL (which they called the plasma mantle). On the other hand, the source region of R2 is thought to be the ring current region, as reviewed by Cowley (2000).

Fujii et al. (1981) examined the seasonal dependence of R0 (which they called the cusp current), R1 and R2 at 08:00-14:00 in MLT using the magnetic field data acquired by the TRIAD satellite. They found that the intensity of $\mathrm{R} 0, \mathrm{R} 1$ and R2 in the summer hemisphere is larger than that in the winter hemisphere. However, they did not distinguish whether the seasonal dependence of R0, R1 and $\mathrm{R} 2$ comes from the ionospheric conductivity (which is season dependent) or some other factors that depend on the season. Fujii and Iijima (1987) found that the R1 current intensity is strongly controlled by the ionospheric conductivity, while the R2 current intensity is weakly controlled: Their study is based on MAGSAT measurements at 04:00-10:00 and 14:00-20:00 in MLT, and they analyzed only the data under geomagnetically quiet conditions, because they estimated the ionospheric conductivity as a function of only the solar illumination, whose function is invalid during 
geomagnetically active times when the conductivity is much affected by particle precipitations. Fujii and Iijima suggested that the above-stated difference between R1 and R2 can be attributed to the different driving mechanisms, that is, $\mathrm{R} 1$ is mainly driven by a voltage source and $\mathrm{R} 2$ is driven by a combination of voltage and current sources. We also note that Fujii and Iijima (1987) did not analyze FACs in 10:00-14:00 MLT segments and R0. On the other hand, Ohtani and Higuchi (2000) showed several cases in which R2 in the winter hemisphere was much smaller than that in the summer hemisphere, suggesting that the intensity of the $\mathrm{R} 2$ current is also controlled by the ionospheric conductance. To summarize, the dependence of the midday FACs (R2, R1 and $\mathrm{R} 0$ ) on the ionospheric conductance has not been fully understood yet.

In this study we statistically examine the dependence of the intensity of dayside FACs on the ionospheric condition, using magnetic field data acquired from the DMSP-F7 satellite and address the source mechanism (a current source or a voltage source) of each FAC system with special attention to the R0 system, which has not been studied in detail in the past. For this purpose, we analyze only the data under geomagnetically quiet conditions, as Fujii and Iijima (1987) did. (The actual procedure to select quiet intervals will be stated in Sect. 2.1.)

\section{Data and method}

DMSP-F7 is a sun-synchronous satellite with a nearly circular orbit at an altitude of $835 \mathrm{~km}$, with its ascending (descending) and descending (ascending) nodes at 10:30 and 22:30 LT (local time) for Northern (Southern) Hemisphere, respectively. In this study we use three-component magnetic field measurements (Rich et al., 1985) made throughout the entire interval of this satellite mission (December 1983 to January 1988). The time resolution of the data is $1 \mathrm{~s}$.

\subsection{Calculation of FAC intensity}

An automatic procedure to identify FAC structures has been developed by Higuchi and Ohtani (2000). The procedure is used to calculate the FAC intensity in this paper. We outline the procedure below. The procedure identifies FAC structures from magnetic field perturbations observed by the DMSP-F7 satellite. DMSP-F7 measures three magnetic field components in spacecraft coordinates. In this coordinate system $\mathrm{X}$ points vertically downward, $\mathrm{Y}$ is in the direction of the satellite velocity projected onto the horizontal plane, and $\mathrm{Z}$ completes a right-hand orthogonal system. We rotate this coordinate system in the Y-Z plane by applying the (two-dimensional) minimum variance analysis (MVA) to $B_{y}$ and $B_{z}$ for the interval during which they show major perturbations, and we obtain $B_{A}$ $\left(B_{L}\right)$ as the magnetic component in the direction of the maximum (minimun) variance. $B_{L}$ is approximately in the "latitudinal" direction (positive poleward (equatorward) in the dayside Northern (Southern) Hemisphere), and $B_{A}$ is approximately in the "azimuthal" direction (positive eastward on the dayside, irrespective of hemispheres). If FACs have a planar sheet structure, the associated magnetic variation should be confined in $B_{A}$. As the satellite crosses a downward-(upward-)flowing FAC sheet, $B_{A}$ increases (decreases) . The procedure by Higuchi and Ohtani fits line segments (polyline) to $B_{A}$ variations measured along a DMSP orbital segment between the pole and the equator, and puts out the optimal number of the fitted line segments. We exclude the most poleward and the most equatorward segments and consider the remaining segments as the FAC sheet crossings. Each of the remaining segments is connected at its edges to adjacent segments; we call the joint points the nodes. Each of the remaining segments is also associated with a value of the $B_{A}$ variation, which characterizes the FAC intensity flowing over the segment: By assuming each FAC sheet is infinitely elongated in both longitudinal and vertical directions, we can calculate FAC intensities from the $B_{A}$ variation according to Ampere's law, that is, $\mu_{0} I=B_{A}$, where $I$ is the FAC intensity $(\mathrm{A} / \mathrm{m})$. The polarity of $B_{A}$ variation gives us the direction of the FAC.

The success of the segment fit can be evaluated by two parameters, $R_{\mathrm{fit}}$ and $\alpha$. $R_{\mathrm{fit}}$ is the measure of the fitness of the polyline: $R_{\mathrm{fit}}=\sqrt{(\mathrm{RSS} / N)} / H_{\mathrm{MAX}}$, where $\mathrm{RSS}=\sum_{i=1}^{N}\left(B_{A}(i)-B_{A \_M O D E L}(i)\right)^{2}, \quad N$ is the number of data, $B_{A}(i)(i=1, \ldots, N)$ is the above-stated observation, $B_{A_{-} \text {MODEL }}(i)(i=1, \ldots, N)$ is the above-stated polyline model fitted to $B_{A}$, and $H_{\mathrm{MAX}}$ is the maximum amplitude of the fitted segments; $H_{\mathrm{MAX}}$ is used here to normalize the event-to-event differences in the $B_{A}$ amplitudes. Lower $R_{\text {fit }}$ means a better fitting. The parameter $\alpha$ shows the square root of the ratio of the larger to smaller eigenvalues of the MVA. The $\alpha$ parameter is infinite if the FAC has a sheet structure. We use FAC crossings with $R_{\mathrm{fit}}<12$ and $\alpha>1.5$ in this study.

The left panel of Fig. 1 plots $B_{A}$ for an example of a dayside FAC crossing, which was observed in the Northern Hemisphere on 16 April 1986. The thin line plots $B_{A}$, and the thick line show the result of the segment fit by the procedure. There are three segments in this example in association with the FAC crossing and we can identify them as upward R2, downward R1 and upward R0 currents from equatorward to poleward. The corresponding orbital segment of this example is shown in the right panel with grey dots showing the node points of the segment fit.

Higuchi and Ohtani (2000) applied this procedure to the entire set of magnetic field data acquired by the DMSP-F7 satellite during 1339 days and composed a database; we call this database "summary data". We use this database for our study. In addition, as stated in Sect. 1, we use only the data during geomagnetically quiet intervals, which we define, as Fujii and Iijima (1987) did, by $K_{p}<1$ and $|\mathrm{AL}|<50 \mathrm{nT}$. (Here, " $K_{p}$ " and "AL" refer to the $K_{p}$ and AL indices.) 


\section{DMSP-F07}

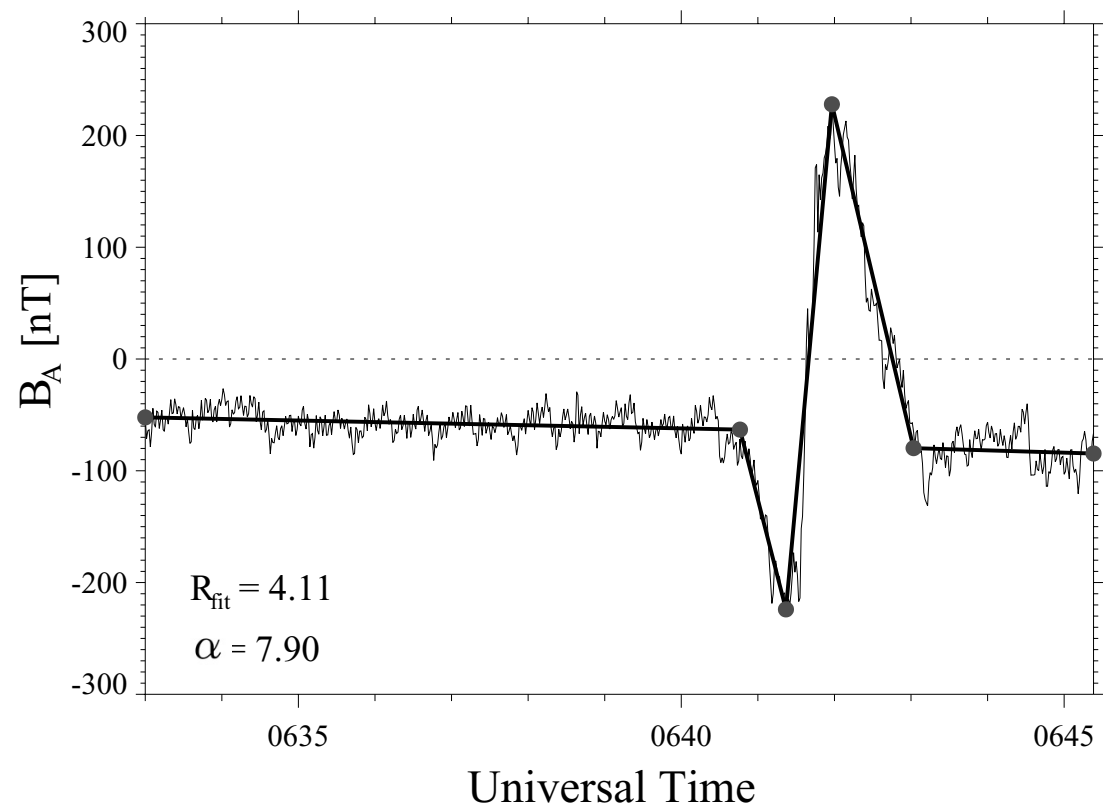

Apr. 16, 1986

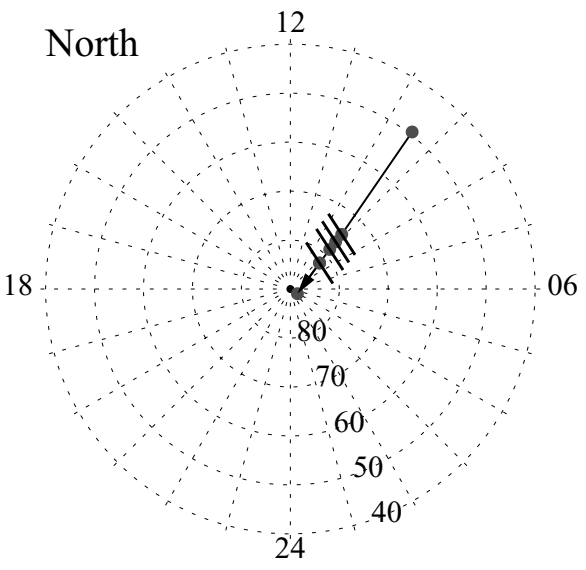

Fig. 1. Left panel: An example of time-domain plot of $B_{A}$ measured by the DMSP-F7 satellite on the dayside in the Northern Hemisphere for the orbit of 16 April, 1986. The thin line shows the raw data of $B_{A}$, and thick line shows the line fitted by the prodecure of Higuchi and Ohtani (2000) (see text for details). The three slopes in this figure correspond to upward R2, downward R1 and upward R0, from left to right. Right panel: Shows the orbit of the path used in the right panel. Grey dots show the node points of the segment fit, and the segments on the four of the dots show the direction of maximum variance in the magnetic field; $B_{A}$ is measured along the segments.

\subsection{Ionospheric conductivity models}

The ionosphere is mainly ionized in two ways, solar EUV and high-energy particle precipitation. According to Mehta (1978), the effect of the latter is negligible under quiet geomagnetic conditions; that is, Mehta has shown that under quiet conditions $\left(K_{p}=0^{+} \sim 2^{+}\right)$, which meant the average $\mathrm{AE}$ of $82 \mathrm{nT}$ for his data set), dayside ionospheric conductivity depends only on the solar zenith angle, in the following mathematical form:

$\Sigma_{P}=12.579-0.112 \chi$

where $\chi$ in degrees is the absolute value of the solar zenith angle, and $\Sigma_{P}$ in mho is the ionospheric Pedersen conductivity. Mehta used the data of $45^{\circ} \leq \chi \leq 95^{\circ}$ to obtain this Eq. 1. We adopt two assumptions for our analysis:

1. The conductivity is constant about $\chi$ and is equal to $\Sigma_{P}\left(95^{\circ}\right)=1.939$ mho for $\chi>95^{\circ} \quad$ (Fujii and Iijima (1987) also used this assumption);

2. This formula can be extended to $\chi \leq 45^{\circ}$. (This assumption is required because the minimum value of $\chi$ in our database is 38.65 .)

As stated in Sect. 2.1, our statistical study uses data only during intervals with $K_{p}<1$ and $|\mathrm{AL}|<50$; under this condition, we used the above equation to estimate $\Sigma_{P}$ for each FAC sheet from $\chi$ at the central latitude of the FAC sheet. This procedure to calculate the ionospheric conductivity is essentially the same as was used by Fujii and Iijima (1987). We also note that $\chi$ used here is not the solar zenith angle at the satellite altitude $(835 \mathrm{~km})$ but that at an altitude of $110 \mathrm{~km}$ traced along the field line from the satellite; we have used the AACGM (Altitude Adjusted Corrected Geomagnetic) coordinates (Gustafsson et al., 1992) for the field line tracing.

Moen and Brekke (1993) presented a different formula to express the $\chi$-dependence of $\Sigma_{P}$ in mho, that is,

$\Sigma_{P}=S a^{0.49}\left(0.34 \cos \chi+0.93 \cos ^{1 / 2} \chi\right)$,

where $S_{a}$ is the $10.7 \mathrm{~cm}$ solar flux index. We will use this model, too, in the following to estimate the ionospheric conductivity.

\subsection{Classification of FAC}

We use paths for which two- or three-sheet structures were observed, and we classify the FACs into a set of R2 and $\mathrm{R} 1$ (R2, R1 and R0) from equatorward to poleward for the two (three) sheet structures. Here we exclude currents if their polarities are opposite to the expected ones, which are downward (upward) for R1 (R0 and R2). We also exclude FAC crossings if the satellite entry and exit points of each FAC are separated by more than $1 \mathrm{~h}$ in MLT, because for such 
crossings, the variation of $B_{A}$ may reflect the longitudinal structure of FACs.

We focus on FACs in the 08:00-12:00 MLT sector in this study. Because of the DMSP-F7 orbital characteristics, there are too few events in other MLT sectors. For this MLT sector we have identified $304(535,584)$ events for R0 (R1, R2) current crossings. Figure 2 shows DMSP-F7 orbital segments analyzed in this paper. Both northern passes and southern passes are plotted in this figure (for southern passes, the signs of their latitudes are reversed). Note that if all orbits are plotted, they cover wider MLTs. We will apply two-hour binning to these data, like Fujii and Iijima (1987) did to their data.

\subsection{Current source and voltage source}

According to the generator models of Fujii and Iijima (1987), a current source (voltage source) leads to a relationship between the FAC intensity and the northern/southern ionospheric conductivity, as shown in the upper-left (right) panel of Fig. 3 (the same as Fig. 1 of Fujii and Iijima (1987)). The ionospheric conductivity is normalized by a typical value of the conductivity without solar illumination, which is much smaller than the dayside ionospheric conductivity. The intensity of a voltage-driven current is proportional to the ionospheric conductivity, but that of the current-driven current is not. Fujii and Iijima assumed that FACs in the Northern and Southern Hemispheres share the same source (i.e. FACs are flowing on closed field lines) for these models. However, this is not the case if FACs flow on open field lines (Erlandson et al., 1988; Bythrow et al., 1988; Ohtani et al., 1995).

Fujii and Iijima (1987) used the equation

$I_{N}+I_{S}=C$ (constant)

for the current generator, where $I_{N}\left(I_{S}\right)$ is the current intensity for the Northern (Southern) Hemisphere. Here the ratio of $I_{N}$ and $I_{S}$ is determined by the ratio of the ionospheric conductivities in Northern and Southern Hemispheres, thus Eq. (3) leads to a weak but not linear correlation. On the other hand, as to FACs flowing on open field lines, we do not have to think about two hemispheres, that is,

$I_{N}=C \quad I_{S}=C^{\prime}$.

In this case the FAC intensity has no correlation with the ionospheric conductivity. For the voltage generator, the same discussion holds as to whether the FAC is flowing on open or closed field lines and the FAC intensity should have a linear correlation with the ionospheric conductivity, that is,

$$
I_{N}=K \times \Sigma_{P, N} \quad I_{S}=K^{\prime} \times \Sigma_{P, S},
$$

where $\Sigma_{P, N}\left(\Sigma_{P, S}\right)$ is the ionospheric Pedersen conductivity for Northern (Southern) Hemisphere. The lower-left (right) panel of Fig. 3 shows a relationship between the FAC intensity and the ionospheric conductivity for FACs driven

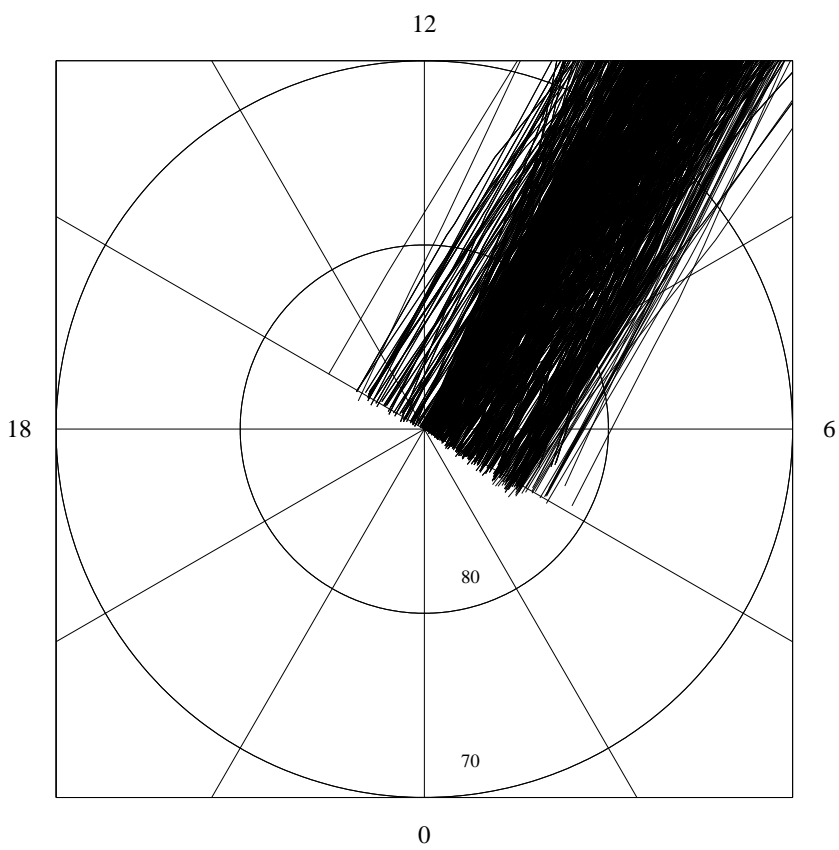

Fig. 2. Orbital segments of DMSP-F7 satellite along which two- or three-sheet structures were observed in the Northern and Southern Hemisphere.

by current source (voltage source) flowing on open field lines. Thus, we can address the source mechanism of a FAC system by examining the dependence of its intensity on the ionospheric conductance.

\section{Results}

Figures $4 \mathrm{a}(4 \mathrm{~b}, 4 \mathrm{c})$ plots $B_{A}$ variations associated with the crossings of R0 (R1, R2) currents versus the ionospheric conductivity for the 08:00-10:00 MLT and 10:00-12:00 MLT bins in the left and right panels, respectively. FAC intensities corresponding to $B_{A}$ variations are labeled on the right axis. Mehta's formula is used for the calculation of the ionospheric conductivity in this figure. $\mathrm{R} 1$ currents are flowing into the ionosphere, and R0 and R2 currents are flowing out of the ionosphere in both MLT sectors. The sign of the $B_{A}$ displacement of R0 and R2 is reversed for easy comparisons with $\mathrm{R} 1$.

We have fewer events in 10:00-12:00 MLT than in 08:00-10:00 MLT because the number of satellite orbits in 11:00-12:00 MLT is small and, as stated in Sect. 2.3, although DMSP-F7 observed both prenoon-type and postnoon-type FACs at 10:00-12:00 MLT (Erlandson et al., 1988), we have excluded postnoon-type FACs. We also have fewer R0 currents than R1 or R2 currents because R0 is included only in the three-sheet structure; in contrast, R1 and R2 currents, can be found for both two- and three- sheet structures.

In both MLT bins and for all current systems, the current intensity tends to increase with an increase in the 

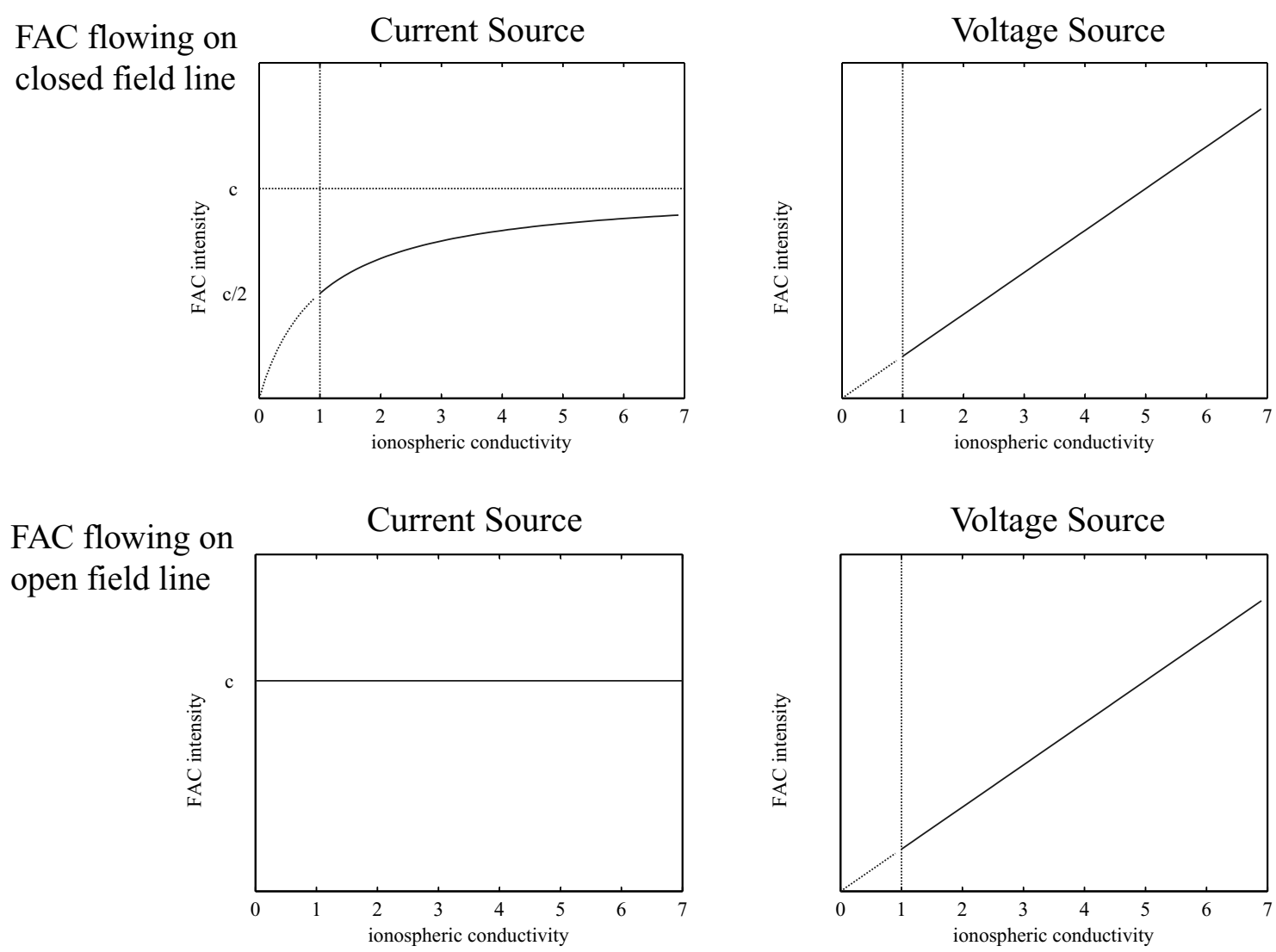

Fig. 3. The relationship between the FAC intensity and the ionospheric conductivity for a current source case and a voltage source case. We assume FAC flowing on closed (open) field lines in upper (lower) two panels.

Table 1. The correlation coefficients between the FAC intensity and the ionospheric conductivity.

\begin{tabular}{lllll}
\hline & \multicolumn{2}{c}{ Using Mehta's equation } & \multicolumn{2}{c}{ Using Moen and Brekke's equation } \\
\hline & MLT 08:00-10:00 & MLT 10:00-12:00 & MLT 08:00-10:00 & MLT 10:00-12:00 \\
\hline R0 & $0.455(0.405 \sim 0.507)$ & $0.768(0.728 \sim 0.816)$ & $0.515(0.471 \sim 0.557)$ & $0.803(0.773 \sim 0.837)$ \\
\hline R1 & $0.607(0.580 \sim 0.637)$ & $0.752(0.713 \sim 0.797)$ & $0.629(0.602 \sim 0.656)$ & $0.784(0.753 \sim 0.818)$ \\
\hline R2 & $0.249(0.216 \sim 0.288)$ & $0.464(0.398 \sim 0.527)$ & $0.266(0.233 \sim 0.303)$ & $0.464(0.399 \sim 0.528)$ \\
\hline
\end{tabular}

conductivity. It is noted that the conductivity dependence of the intensity of R1 is larger than that of R2 and R0; stated differently, for a fixed value of the conductivity, the current intensity of R1 looks larger than that of R0 and R2. If we look at the example used in Fig. 2, its $B_{A}$ variations (the FAC intensities) are $160.86(0.13), 451.94(0.36)$ and $307.64 \mathrm{nT}$ $(0.24 \mathrm{~A} / \mathrm{m})$ for $\mathrm{R} 2, \mathrm{R} 1$ and $\mathrm{R} 0$. This may be related to the balance of dayside FACs, which we will discuss in the next section.

We note that the correlation between the current intensity and the conductivity is different for different current systems. In Fig. 4 the R1 (panel (b)) and R0 (panel (a)) currents look to be more correlated with the ionospheric conductance than the
R2 current (panel (c)). Table 1 lists the (linear) correlation coefficients (including their statistical errors) between the conductivity and the current intensity for those three current systems in both MLT sectors. We have used the bootstrap method (Efron and Tibshirani, 1993) for the error estimation. We did the same analysis using Moen and Brekkes' formula and the result is also listed in Table 1, which shows that Moen and Brekkes' formula gives a better correlation than Mehta's formula for all FAC systems and both MLT sectors, but the difference among the current systems is essentially the same as the result based on Mehta's formula. Figure 5 plots the correlation coefficients and their estimated errors for R0, R1 and R2 from the left; the stars show the correlation 
[a]

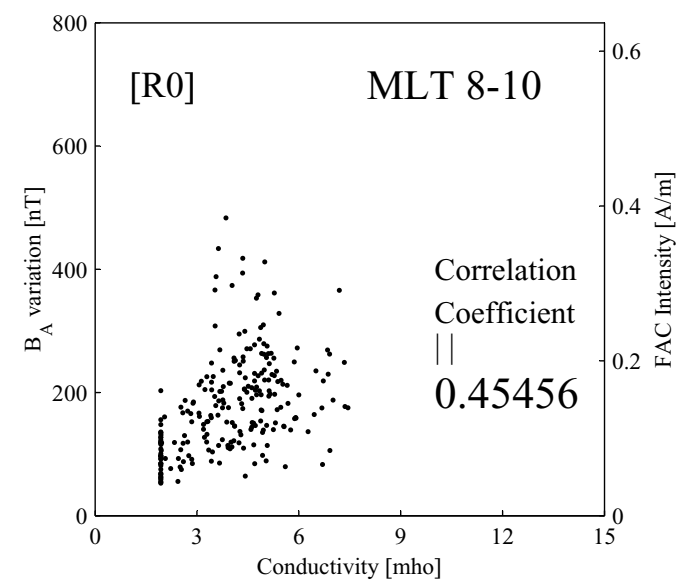

[b]

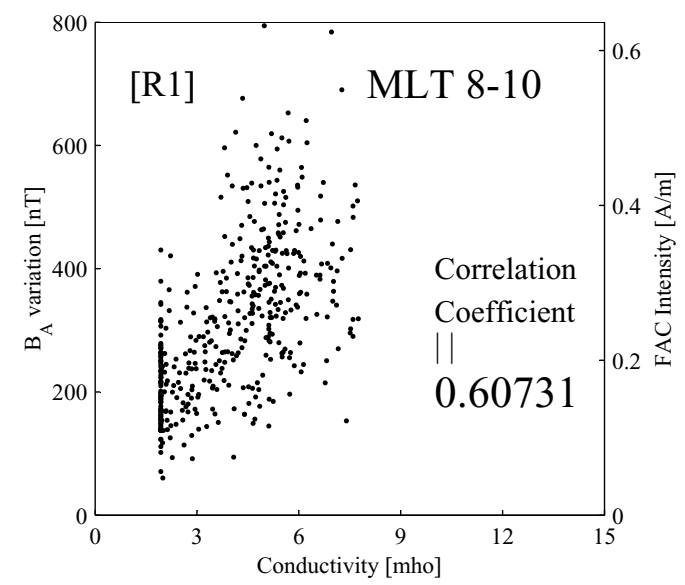

[c]

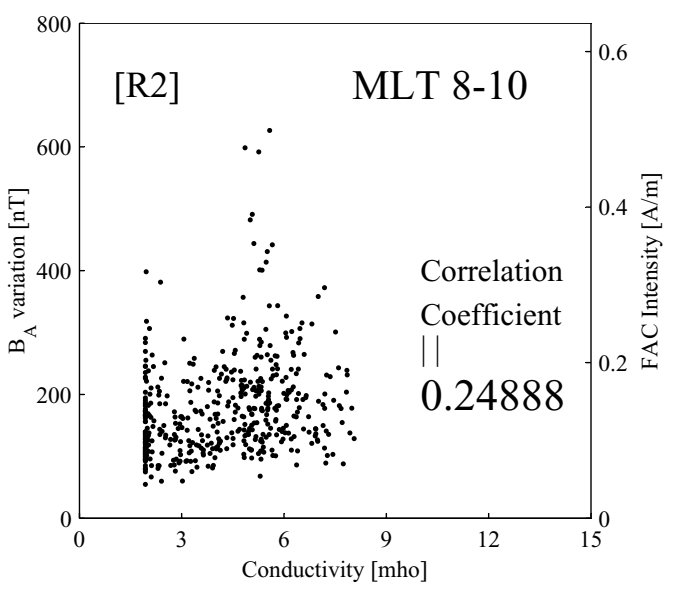

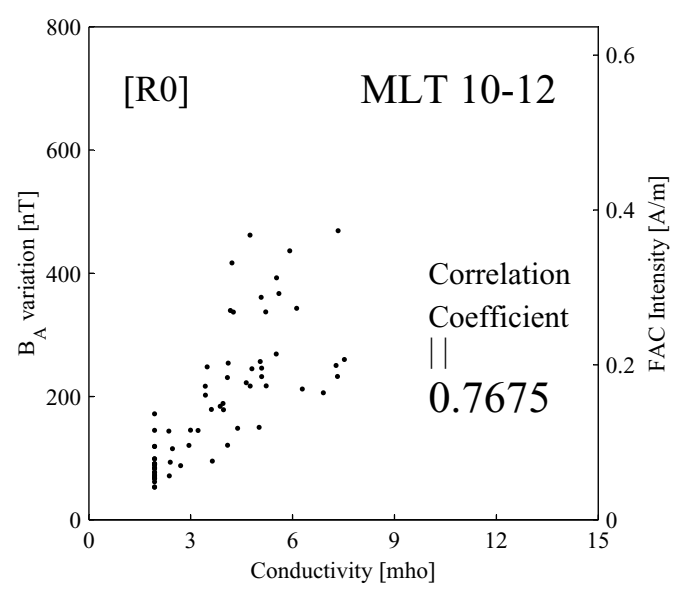
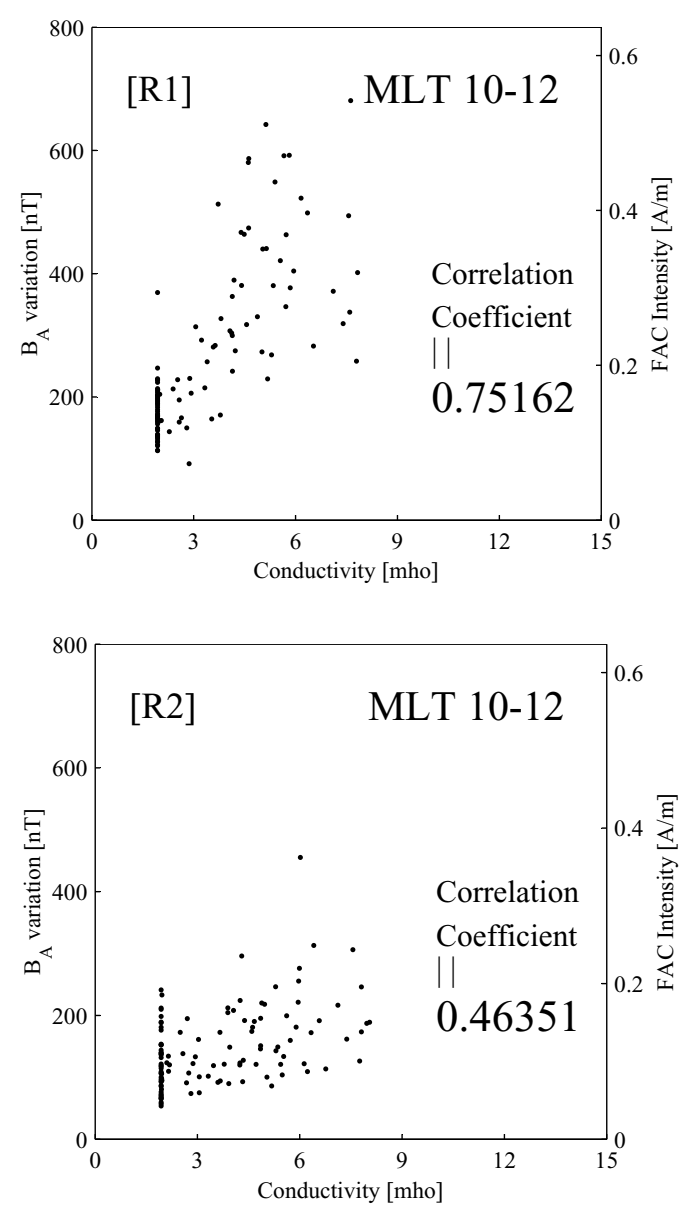

Fig. 4. Scatter plots of the $B_{A}$ displacement versus the ionospheric conductivity in each two-hour MLT bin from 08:00-12:00 MLT. The top (middle, bottom) panel shows the plot for the data of R0 (R1, R2).

coefficients from Mehta's formula and the vertical line segments show the error bars. The correlation coefficient of R1 is significantly larger than that of R2 in both MLT sectors. For R0, the correlation coefficient is larger than that of $\mathrm{R} 2$ in both MLT and similar to that of R1 in 10:00-12:00 MLT. In 08:00-10:00 MLT the correlation coefficient of R0 is smaller than that of R1 and larger than that of R2. This result indicates that the intensities of the R1 and R0 currents are more strongly controlled by the ionospheric conductivity than that of the R2 current, that is, the sources of the R1 and R0 systems behave more like voltage generators than the source of the R2 system. We note that the difference between R1 and R2 is consistent with the results of Fujii and Iijima (1987), although the MLT coverage is different. 

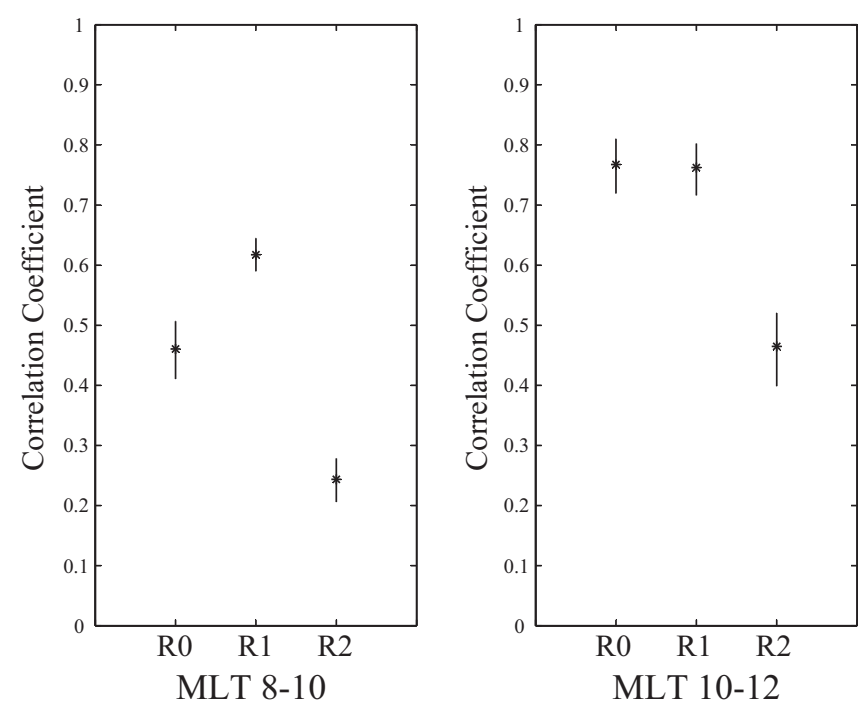

Fig. 5. Shows the correlation coefficient between the $B_{A}$ displacement and the ionospheric conductivity calculated from Mehta's equation for R0, R1 and R2 for the 8-10h (left panel) and 10-12 h (right panel) MLT bins. The vertical segment around the star shows the error bar calculated by using the bootstrap method.

On the other hand, our result of the dependence of the R0 current intensity on the solar zenith angle is consistent with the result of Fujii et al. (1981), who found that the intensity of R0 in the summer hemisphere is larger than that in the winter hemisphere. Our results confirm that the seasonal dependence reported by Fujii et al. (1981) comes from the seasonal dependence of the ionospheric conductivity.

\section{Discussion}

As we have shown in Fig. 5, the current intensity of R1 is better correlated with the ionospheric conductivity than that of R2 under geomagnetically quiet conditions. This suggests that the generator of the R1 current is more like a voltage source (Sect. 1). On the other hand, R2 is more consistent with the idea that this current system is driven by a current source, which provides a constant current to the ionosphere irrespective of the ionospheric conductivity. These tendencies are consistent with the results of Fujii and Iijima (1987), although the MLT coverage is different. Figure 5 also shows that the current intensity of R0 is correlated with the ionospheric conductivity significantly better than that of R2, suggesting that the generator of the $\mathrm{R} 0$ current is also a voltage source. This conclusion may be interpreted in terms of the closure of R0 and R1 currents at the high-latitude tail boundary, where the kinetic energy of the solar wind (magnetosheath) flow can be converted to the electromagnetic energy as the flow is decelerated (see Fig. 11 of Ohtani et al., 1995); because the driving voltage of this generator comes from the solar wind velocity and the magnetic field (i.e. $\mathrm{E}=-\mathrm{V} \times \mathrm{B}$ ), this voltage is determined irrespective of the condition of the ionosphere; this generator is therefore thought to be voltage-driven. The idea of this voltage generation was originally proposed by Stern (1984) for the generation of the R1 current.

Christiansen et al. (2002) showed that during quiet periods dayside FACs are closed at a given LT in all seasons, that is, irrespective of the solar zenith angle. This is, at least for two sheet structure FAC, inconsistent with our results that the solar zenith angle dependence of the intensity of R1 is more pronounced than that of $\mathrm{R} 2$. (For example, if $\mathrm{R} 1$ is balanced with R2 when the solar zenith angle is $80^{\circ}, \mathrm{R} 1$ cannot be balanced with $\mathrm{R} 2$ when the solar zenith angle is $50^{\circ}$, since the R1 intensity increases more sharply than the R2 intensity with increasing solar zenith angle.) Thus, we have examined the balance of dayside FACs. The result shows that for the two sheet structure events, the average of the net current in the 08:00-12:00 MLT sector is $0.053 \pm 0.007 \mathrm{~A} / \mathrm{m}$ $\left(67.51 \pm 9.65 \mathrm{nT}\right.$ in $\left.B_{A}\right)$, that is, the average net current is significant. On the other hand, for the three-sheet-structure events, the result is $0.0010 \pm 0.0034 \mathrm{~A} / \mathrm{m}(-1.25 \pm 4.33 \mathrm{nT}$ in $B_{A}$ ), that is, the net current does not exist statistically. We have also found that the net current intensity of the two-sheet-structure events is correlated with the solar zenith angle (correlation coefficient is 0.52), while that of the three-sheet-structure events is not (correlation coefficient is 0.054). Therefore, our result for the three-sheet-structure events is consistent with the results of Christiansen et al. (2002), but our result for the two-sheet-structure events is not.

These results may be interpreted by considering that the R1 system has actually two sources, one being current-driven (c-R1 below), which is closed with R2, and the other being voltage-driven (v-R1 below). The v-R1 current should flow in the poleward region of $\mathrm{c}-\mathrm{R} 1$. For a three-sheet FAC structure, v-R1 is considered to be closed with R0 at a given MLT, and therefore, no net current exists. On the other hand, for a two-sheet FAC structure, v-R1 should make the net current. The left (right) panel of Fig. 6 illustrates the current closure of dayside FACs when they have the two-sheet (three-sheet) structure. For the two-sheet case (left panel), the ionospheric closure of the net current (=v-R1) still remains to be understood, but one possibility is that it is closed with $\mathrm{v}-\mathrm{R} 1$ in the postnoon sector or $\mathrm{R} 0$ at different MLTs.

Watanabe et al. (1998) studied (R0, R1) pairs (with no $\mathrm{R} 2$ observed) and found that R0 and R1 had almost the same intensities, and they suggested that the two currents are closed latitudinally. Their (R0, R1) current pair can be regarded as (R0, v-R1) in our terminology, because the intensities of R0 and $\mathrm{v}-\mathrm{R} 1$ are the same; in their events the (c-R1, R2) pair was presumably nonexistent. Some papers suggested that there is no R2 in the midday sector (Iijima and Potemra, 1978; Erlandson et al., 1988) and it is interesting to note that the midday R1 may be constructed by v-R1 only.

Some studies reported that R1 includes the open-close boundary (Xu and Kivelson, 1994, Ohtani et al., 2000). It is possible that $\mathrm{v}-\mathrm{R} 1$ mainly flows in the open field line region 


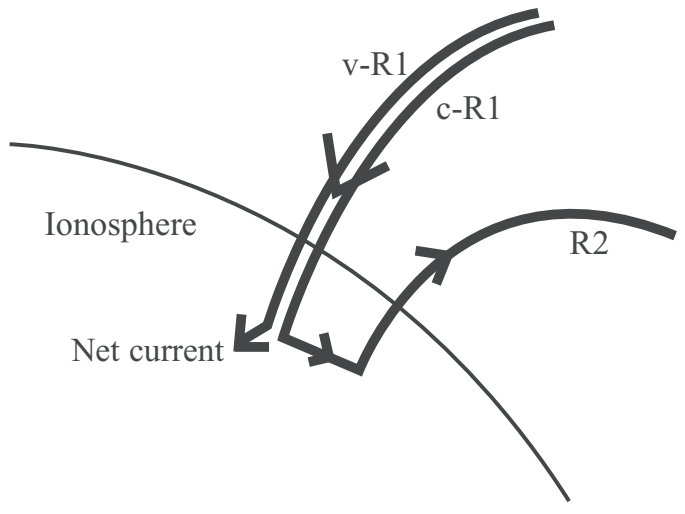

Two-sheet structure FACs

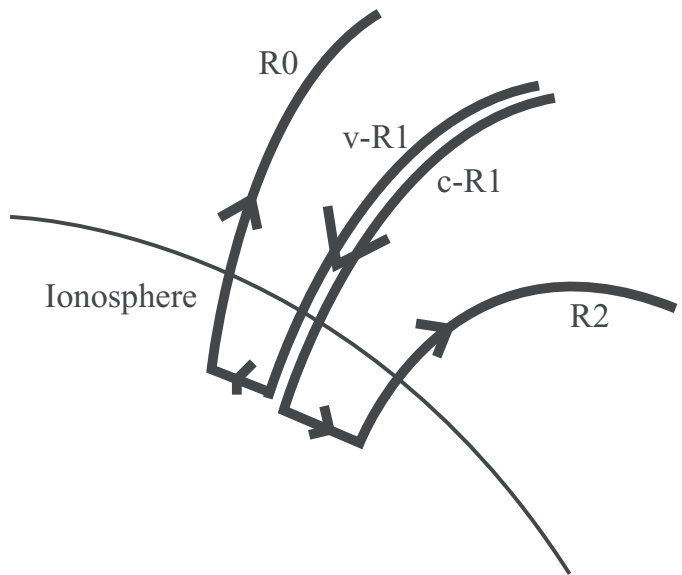

Three-sheet structure FACs

Fig. 6. Illustrates the ionospheric current closure for two-sheet (three-sheet) structure FACs on the left (right) panel.

while c-R1 mainly flows in the closed field line region. The source of the R1-R0 pair in Ohtani et al. (1995) (Fig. 11 in their paper) was assumed to exist on open field lines, thus it may correspond to the source of $\mathrm{v}-\mathrm{R} 1$.

\section{Summary}

We have investigated the correlation between the ionospheric conductivity and the FAC intensity in the dayside (MLT=08:00-12:00) region using DMSP-F7 magnetometer data. The results are summarized as follows:

1. In the dayside region (MLT=08:00-12:00), R1 intensity has a higher ionospheric conductivity dependence than R2 intensity, suggesting that R1 is associated with the voltage-like source and R2 is associated with a more current-like source. This is consistent with the results of Fujii and Iijima (1987), who studied dawn (MLT=04:00-10:00) and dusk (MLT=14:00-20:00) FACs.

2. In the same MLT range, R0 intensity also has higher ionospheric conductivity dependence than R2 intensity, suggesting that $\mathrm{R} 0$ is associated with a voltage-like source.

3. Dayside FAC intensities are latitudinally well balanced when they have a three-sheet-structure (R0, R1 and R2); on the other hand for a two-sheet-structure (R1 and R2), the intensity of R1 is larger than that of R2 so that the net current has the polarity of R1.

These new results strongly support the coupling of R0 and $\mathrm{R} 1$ in the magnetosphere, that is, $\mathrm{R} 0$ and a part of $\mathrm{R} 1$ are thought to be created by the interaction between the solar wind and the magnetic field of the Earth in the open field region. We also note that R1 may consist of two systems, that is, $\mathrm{v}-\mathrm{R} 1$ and c-R1 having the characteristics of a voltage source and a current source, respectively.

Acknowledgements. The DMSP-F7 magnetometer data were provided by F. J. Rich. The data of $\mathrm{AL}$ and $K_{p}$ index were provided by World Data Center. This research was partially supported by the Ministry of Education, Culture, Sports, Science and Technology, Grant-in-Aid for Scientific Research (B), 12558023 and (A), 14208025. Work at APL was supported by the NSF grant ATM-9901102 and the NASA grant NAG5-10941.

Topical Editor M. Lester thanks a referee for his help in evaluating this paper.

\section{References}

Bythrow, P. F., Potemra, T. A., Erlandson, R. E., and Zanetti, L. J.: Birkeland currents and charged particles in the high-latitude prenoon region: A new interpretation, J. Geophys. Res., 93, 9791, 1988.

Christiansen, F., Papitashvili, V. O., and Neubert, T.: Seasonal variations of high-latitude field-aligned currents inferred from Orsted and MAGSAT obserbations, J. Geophys. Res., 107, A2, 10.1029/2001JA900104, 2002.

Cowley, S. W. H.: ISEE magnetosphere-ionosphere interactions: A tutorial review, Magnetospheric Current Systems Geographical Monograph 118, 91, 2000.

Efron, B. and Tibshirani, R. J.: An introduction to the bootstrap, Chapman \& Hall, New York, 1993.

Erlandson, R. E., Zanetti, L. J., Potemra, T. A. Bythrow, P. F., and Lundin, R.: IMF $B_{y}$ dependence of region 1 Birkeland currents near noon, J. Geophys. Res., 93, 9804, 1988.

Fujii, R., Iijima, T., Potemra, T. A., and Sugiura, M.: Seasonal dependence of large-scale Birleland currents, Geophys. Res. Lett., 8, 1103, 1981.

Fujii, R. and Iijima, T.: Control of the ionospheric conductivities on large-scale Birkeland current intensities under geomagnetic quiet conditions, J. Geophys. Res., 92, 4505, 1987. 
Gustafsson, G., Papitashvili, N. E., and Papitashvili, V. O.: A revised corrected geomagnetic coordinate system for Epochs 1985 and 1990, J. Atmos. Terr. Phys., 54, 1609, 1992.

Higuchi, T. and Ohtani, S.: Automatic identification of large-scale field-aligned current structures, J. Geophys. Res., 105, 25305 , 2000.

Iijima, T. and Potemra, T. A: Large-scale characteristics of field-aligned currents associated with substorms, J. Geophys. Res., 83, 599, 1978.

Mehta, N. C.: Ionospheric electrodynamics and its coupling to the magnetosphere, Ph.D. thesis, Univ. of Calif., San Diego, 1978.

Moen, J. and Brekke, A.: The solar flux influence on quiet time conditions in the auroral ionosphere, Geophys. Research Letter, 20, 971, 1993.

Ohtani, S., Potemra, T. A., Newell, P. T., Zanetti, L. J., Iijima, T., Watanabe, M., Yamauchi, M., Elphinstone, R. D., de la Beaujardiere, O., and Blomberg, L. G.: Simultaneous prenoon and postnoon observations of three field-aligned current systems from Viking and DMSP-F7,J. Geophys. Res., 100, 119, 1995.

Ohtani, S. and Higuchi, T.: Four-sheet structures of dayside field-aligned currents: Statistical study, J. Geophys. Res., 105, $25317,2000$.
Ohtani, S., Higuchi, T., Sotirelis, T., and Newell, P. T.: Disappearance of large-scale field-aligned current systems: Implication for the solar wind-magnetosphere coupling, Magnetospheric Current Systems Geographical Monograph 118, 253, 2000.

Rich, F. J., Hardy, D. A., and Gussenhoven, M. S.: Enhanced ionosphere-magnetosphere data from the DMSP satellites, Eos Trance. AGU, 66, 513, 1991.

Siscoe, G. L., Lotko, W., and Sonnerup, B. U. Ö.: A high-latitude, law-latitude boundary layer model of the convection current system, J. Geophys. Res., 96, 3487, 1991.

Stern, D. P.: Magnetospheric dynamo processes, Magnetospheric Currents, Geophys. Monograph Ser., Vol. 28, edited by Potemra, T. A., AGU, Washington D.C., 200, 1984.

Watanabe, M., Iijima, T., Nakagawa, M. Potemra,, T. A., Zanetti, L. J., Ohtani, S., and Newell, P. T.: Field-aligned current systems in the magnetospheric ground State, J. Geophys. Res., 98, 6853, 1998.

$\mathrm{Xu}$, D. and Kivelson, M. G.: Polar cap field-aligned currents for southward interplanetary magnetic fields, J. Geophys. Res., 99, 6067, 1994. 